\title{
Internet of Things key technologies and architectures research in information processing
}

\author{
Shi Yan-rong \\ Electronic Information Department \\ Xi’an Railway Vocational \& Technical Institute \\ Xi'an, China \\ E-mail: shishiyr@126.com
}

\begin{abstract}
The Internet of things is the key strategic application in the twelfth Five-Year plan of emerging technology industries in China. This paper introduces the basic concepts of Internet of things, proposes a three-layer model development platform based on the International Telecommunication Union (ITU) specification, and describes the key technology and architecture in the platform, and gives smart home application development based on the platform.
\end{abstract}

Keywords- Internet of Things(IOT), IOT Development Platform, Smart Home

\section{INTRODUCTION}

The Internet of Things is the third wave of information industry after the Internet and mobile communication. Now many countries have spent heavily in-depth study to explore the Internet of Things. China with Germany, United States, Britain and other countries are together to become one of the international leading power. Many of the concepts come from the "Internet of Things", such as IBM's "Smarter Planet" $^{\text {[1] }}$, and the "Experience China" by some Domestic research institutions and local government.

Specifically, the Internet of Things will be a variety of sensing devices, such as radio frequency identification (RFID), infrared sensors, global positioning systems, remote sensing systems, wireless sensor networks, etc..., to be formed a huge network. Most recently, a RFID Ecosystem creates a microcosm for the IoT at the University of Washington. A suite of Web-based tools which can manage personal RFID data and privacy settings is developed ${ }^{[2]}$. It's purpose is to make all items connected to the network to facilitate the identification and management. And it's full use of the new generation of IT technology in all walks of life among the things to "speak out".

At present, the perception of Things countries mostly remain in the conceptual stage of exploration, such as IBM's Smarter Planet has three characteristics:

- First, is to more thorough perception: That is, to take full advantage of perception measurement, capture and transmission of information equipment, systems or processes anytime and anywhere;

- Second, is to more comprehensive interoperability: Refers to the wisdom of the system to work together according to a new way;

- Third is to more intelligent: that is able to use

\author{
Hou Tao \\ College of Electrical and Information Engineering \\ Shanxi University of Science and Technology \\ Xi'an, China \\ E-mail: houtao@sust.edu.cn
}

advanced technology more intelligent and more insight into the world to create new value.

Meanwhile, currently, there are many infrastructures for data collecting. A practical realization of IoT architecture, which is based on 6LoWPAN standard at the University of Padova, is a flexible and expandable infrastructure, allowing easy node management ${ }^{[3]}$.

In August 7, 2009, when Premier Wen Jiabao research in Jiangsu Wuxi, he pay great attention to $R \& D$ center of the micro-nano sensor developed key technology of things, proposed the idea of creating sensor networks in Wuxi, then radiate to all the Nation. Premier Wen Jiabao pointed out that the sensor development should be planning for the future, the core technology should be earlier break out. And the center of the sensor information, or called "Experience China" center, should be as soon as possible to establish.

In fact, the definition for the Internet of Things hasn't be given with any institutions or government organizations now. This paper is not ready to be explored for the concept and business model of the Internet of Things too. The paper refers to the recommendations of the $\mathrm{ITU}^{[4]}$, and combines the application of Chinese security sensing equipment in the field of information processing, and gives a viable development platform architecture of the Internet of Things. With the architecture, software developers can quickly construct their application based on various types of sensors and data sharing of Internet of Things.

This paper's content is organized as follows: Chapter 2 describes the specification recommends given by ITU, and on this basis, describes the four-layer model of Things development platform architecture, and the detail component functions and the use of key technologies in every layer; Chapter 3 gives a typical application of the secondary development of a smart home industry based on the development platform. And finally chapter is the summary and outlook.

\section{THREE-LAYER MODEL OF THINGS DEVELOPMENT PLATFORM}

\section{A. System proposed composition of the ITU's Internet of Things}

ITU recommendations of Things conceptual model consists of five layers:

- Perception layer: The main function of this layer is to obtain the various types of static / dynamic 
information of the real world through various types of sensors and to share with Internet access.

- Access layer: The main function of this layer is to send the perception layer information to the Internet through the various communication networks (such as mobile communication networks, wireless local area network (WiFi) and satellite network etc.

- Internet layer: The main function of this layer is to establish an efficient and reliable infrastructure platform for upper management and large-scale industrial applications with global Internet platform.

- Service management layer: The main function of this layer is to get real-time management and control of vast amounts of information within the cluster server network with super-computing power and to provide a good user interface for the upper layer application.

- Application layer: The main function of this layer is to integrate underlay system function and built up a practical application for all types of industries, such as the ecological environment and natural disaster monitoring, intelligent transportation, heritage conservation and cultural dissemination, fortune medical and health monitoring etc.

\section{B. Things development platform}

The model by ITU basically covers the essential factors of Things to the interoperability of sensors and Internet connectivity, but it does not apply to the actual software applications, such as the OSI Seven-layer model of TCP/IP, the actual implementation is the fourth layer.

And the platform processes the data to a "Converged data"level by integrating the work of analysis and inference on the basis of the sensing data. So this paper presents the application of architecture is three layer: First is the data acquisition layer; Second is the data service layer; Third is the application layer.

\section{1) Framework}

The Framework of the things development platform is shown in Figure 1.

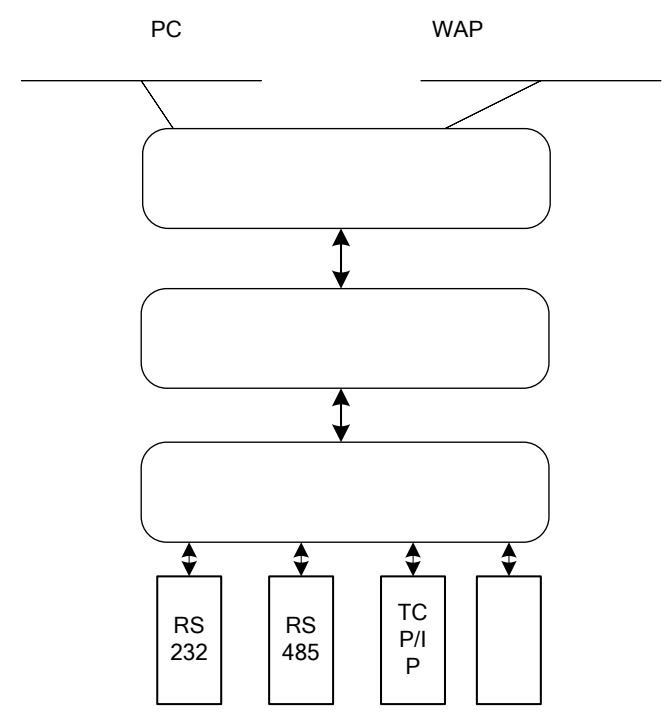

Figure 1 The development platform Framework of IOT

\section{2) Data Acquisition Layer}

The data acquisition layer is equivalent to the perception of the ITU model layer and access layer, complete the following features:

a) Device Driver: The drive system connected to various types of sensor devices, mainly is the Serial UART devices;

b) Data Analysis: Analytical sensor devices to transfer data, and the upper service 's control commands to parse the format for device identification. Currently the most common sensor devices is the RS485/RS232 UART interface, using the MODBUS ${ }^{[5]}$ protocol to transfer data, so the system provides the default drive and data analysis capabilities of such equipment, but if new hardware has different protocol, then developers need to do some extra work to form the dynamic the new link library and add it to the device driver library. MODBUS protocol has become a common industry standard, applied to almost all the electronic controller. It is applied to the communication between the sensor and controller, the controller and the controller;

c) Device Management: The module to manage the different types of sensors connected to the system, to establish the mapping relation between devices, to interact with the equipment and the upper data services layer for the up-link and down-link;

d) Device config: This module provides SOCKET communication interface protocols and interfaces of the IP address / port configuration, including the service layer and the upper data communication. 


\section{3) Data Service Layer}

The data service layer is equivalent to the ITU model of service management, complete the following features:

a) Completion of data collection in SOCKET communication to receive the data from acquisition layer;

b) Providing registration function to the application layer, providing the registration interface by device id or by specific parameters;

c) Pushing the registration data to application layer. The proprietary data is transferred to different users by the registration to reduce network traffic pressure and improve the effective data transfer;

d) Collecting data storage warehousing, the specific storage device and the parameters can be configured;

e) Supporting distributed deployment /interface calls.

\section{4) Application Layer}

The application layer is equivalent to the Internet, and application layers of the ITU model, the main features including:

a) Provide secondary development of the expanded operational capacity, and the open API interface for data registration and device control;

b) Provide the standard WCF interface. WCF's full name is Windows Communication Foundation, likely SOAP PRC web services, it has an interface described in a machine process format (specifically WSDL);

c) The display interface use WPF client technology to support various third-party terminal access, including mobile phone / PC. WPF is Microsoft's new graphics system that provides a unified description and method of operation for the user interface, 2D/3D graphics, documents and media. WPF technology support the the XBAP protocol to deploy application by B/S style for easy access. Between the system interface and data is the distributed structure system. The system can be shown in any type of client, such as PC, mobile device etc.

The data can be classified into two types, which are Nonrealtime Data and Realtime Data, according to its frequency of change and updating. Nonrealtime Data refers to the data which does not change frequently as the time goes, which thus needs not be collected every time a decision is made. As a result, the data can be obtained from both online or offline service providers.

\section{TYPICAL APPLICATION - SMART HOME}

This chapter describes the development process of smart home networking applications instance based on the platform.

\section{A. Smart home system components System components:}

1)Switch control card: the card is to control home lighting and air conditioning equipment with PCI interface, it does not meet the serial port and MODBUS protocol, so we should to re-develop the driver.

2)Serial Device Server: the device is to connect the RFID lockers, smoke sensors and temperature and humidity sensor, it meets the serial port and MODBUS protocol;

3)Data server: It is used to store equipment data, management of the upper service;

4)PC machine: It is used to run the secondary development services to provide real-time data display, voice prompts and historical data query functions.

B. Secondary development process of the smart home

1) Data acquisition layer development

First we develop device driver for the switch control card.

Drivers development in the platform with parameters as below:

Instruct length--Length of the packet transmission

Instruct Type

Instruct Content

CRC check

Different devices have different definitions of specific instruction content, and the switch control car's definition is shown as Table 1:

Table 1 Switch Card protocol

\begin{tabular}{|c|c|c|c|}
\hline $\begin{array}{c}\text { Instruct } \\
\text { length }\end{array}$ & $\begin{array}{c}\text { Instruct } \\
\text { Type }\end{array}$ & Instruct Content & CRC Check \\
\hline 4 Bytes & $10 H$ - Down & $\begin{array}{l}D 0(D 0: 1-8) \\
\text { (0x00(open) or } \\
0 x 01(\text { close }) \text { D1 } \\
\text { (D1: 9-16) } \\
\text { 0--close 1--open } \\
3 \text { bytes }\end{array}$ & 1 Byte \\
\hline 4Bytes & $11 H$-rsp & $\begin{array}{l}\text { D0 (D0: } 1-8) D 1 \\
\text { (D1: } 9-16) \\
0 \text {--close 1--open } \\
2 \text { bytes }\end{array}$ & 1 Byte \\
\hline
\end{tabular}

\section{2) Application layer development}

Business processes are realized by the smart home including:

(1)When received the data of user credit card to home, the system first play the current temperature and humidity data to the user;

(2)The system determines the current temperature and light-sensitive data, if the temperature is higher than the set threshold, the down stream control data is sent to open the air-conditioning equipment, if the sense data of light is less than the set threshold, then open the lighting equipment;

(3)The data acquisition layer receive the upper command and parse the data formats for the device to identify the device control.

\section{SUMMARY AND OUTLOOK}


The application of things is still in an exploratory stage. Compared with the traditional areas of security, Internet of things applications is reflected with intelligence and interaction. And the basis of intelligent and interactive is information processing. Research institutions of all parties have basically reached a consensus at this point.

Some so-called Internet of Things equipment present in the Shanghai Expo, such as the refrigerators of Things, irons and other of Things. But all of them are basically still stuck in the level of perception. How to perceive the combination of things and the Internet intelligent information processing and the sharing is the field of things even broader and more social significance.

The development platform model proposed in this paper combines the ITU specification and the current security system architecture for quick development and deployment of Internet of Things in the field of information-sharing applications. Follow-up work can be further introduced to the experts in the field of data mining, artificial intelligence, knowledge, and thus to extract more valuable information and applications.

\section{REFERENCES}

[1]IBM. Smarter Planet Win in China. http://www-900.ibm.com 2008-11-06;

[2]CASTELLANI A P, BUI N, CASARI P, et al. Architecture and Protocols $\mathrm{f}$ or the Internet of Things: a Case Study [C]. Proceedings of the 8th IEEE International Pervasive Computing and Communications Workshops: Mar 29Apr 2, 2010, Mannheim, Germany . Piscataway , NJ, USA: IEEE, 2010: 678-683;

[3] A WELBOURNE E, BATTLE L, COLE G, et al. Building the Internet of Things Using RFID[J]. IEEE Internet Computing, 2009,13(3):48-55;

[4]ITU NGN-GSI Rapporteur Group. Requirements for support of USN applications and services in NGN environment [R] . Gene-va:International Telecommunication Union ( ITU) ,2007;

[5] GB-Z 19582.1-2004 Based on Modbus Industrial Automation Network Specification. 